\title{
Psychomotor reminiscence as a function of gonadal steroid hormone variation
}

\author{
KAREN C. WELLS \\ Western Psychiatric Institute, University of Pittsburgh School of Medicine, Pittsburgh, Pennsylvania 15260 \\ and
}

R. B. PAYNE

University of Georgia, Athens, Georgia 30602

\begin{abstract}
Psychomotor reminiscence was studied in relation to endogenous variations in estrogenic hormone levels. Forty-three young adult females monitored their menstrual cycles for two replications, then reported for testing on Day 2, 8, or 14 of their cycles. Results showed significantly higher reminiscence and postrest performance on Day 14 (high estrogen) than on the other days (low estrogen). The well-known response activation properties of the estrogens may fulfill one of Hull's (1943) conditions for the generation of reactive inhibition $\left(\mathrm{I}_{R}\right)$, of which reminiscence is regarded as a measure. Since female levels of estrogen are substantially higher than male levels, even at the nadirs of their estrogen cycles, one arrives at a plausible account of the often-demonstrated female dominance in psychomotor reminiscence proclivities.
\end{abstract}

Research on sex, age, and task factors in psychomotor reminiscence has raised new and provocative interpretational issues. For example, in rotary pursuit and mirror tracking tasks, it is now clear that young adult females reminisce more than males of comparable age (Buxton \& Grant, 1939; Huang \& Payne, 1975; Payne \& Huang, 1977), while prepubescent females reminisce less than males of comparable age (Horn, 1975; Zegiob \& Payne, 1977). Further, inasmuch as the experimental operations involved in reminiscence studies are basically like those involved in distributed practice studies, corresponding inversions of sex dominance should be found in practice distribution effects, and they are (McCaffrey \& Payne, 1977; Resick \& Payne, 1978). By contrast, no sex difference in reminiscence has been found at either age level on the reversed-alphabet printing task (Archer \& Bourne, 1956; Huang \& Payne, 1975; Zegiob \& Payne, 1977). Thus, sex dominance appears to be some function of the nature of the task and the developmental status of the subject.

Given the theoretical history of psychomotor reminisecence, an appropriate question is whether the facts of sex dominance and its developmental inversion can be embraced by Hull's (1943) theory of reactive inhibition $\left(I_{R}\right)$. Hull accounted for reminiscence and intertrial interval effects in terms of a temporary responseproduced inhibitory factor $\left(I_{R}\right)$ that accumulated as some function of the frequency and effortfulness of responding and subsided spontaneously as an exponential

Requests for reprints should be sent to R. B. Payne, Department of Psychology, University of Georgia, Athens, Georgia 30602. function of the amount of rest. Further, $I_{R}$ was accorded the status of a primary negative drive, one function of which was to suppress the level of responding. Within this context, McBride and Payne (1979) explored the hypothesis that young adult females reminisce more than males because they accumulate more $I_{R}$ during the prerest trials, with rates of subsidence remaining essentially the same. Sixty males and 60 females practiced continuously for 3 min on a mirror tracking task, then rested for $0,1,2,4$, or $8 \mathrm{~min}$ prior to resumption of massed practice. The data showed unequivocally that (a) reminiscence for both sexes was a negatively accelerated function of the length of the rest period, as Ammons (1947), Irion (1949), and Kimble and Horenstein (1948) found for sexually undifferentiated samples; (b) the asymptotic level of reminiscence was significantly higher for females; (c) the sexes approached their asymptotic levels at comparable rates; and (d) the gain per unit of prerest practice was significantly smaller for females. Thus, a good match between data and theory was obtained, although it remains to be seen whether comparable agreement can be found at the prepubescent level.

The obvious question, of course, is why young adult females are more susceptible than males to the decremental effects of massed practice. Although several hypotheses might be advanced, for example, differences in muscularity and associated values of Hull's constant "c" (1943, p. 279), the collective facts of sex differences and their inversion across puberty suggested a look at the possible role of gonadal steroid hormones, particularly the estrogens. Endocrinologists have known for a long time that estradiol-17 $\beta$, estrone, and estriol, all 
of which are synthesized and secreted by the human ovary (Nocenti, 1968), possess activating properties manifested in a variety of behaviors (Broverman, Klaiber, Kobayashi, \& Vogel, 1968; Vogel, Broverman, \& Klaiber, 1971). The estrogens, like the amphetamines, lower arousal thresholds to environmental stimuli (Kawakami \& Sawyer, 1967) and enhance central adrenergic processes, perhaps because they inhibit monoamine oxidase activity (Vogel et al., 1971). Among lower animals, such as the white rat, ovarian estrogens and their metabolites stimulate running activity and restore this activity in ovariectomized animals (Young \& Fish, 1945; Gerall, Napoli, \& Cooper, 1973). Wooley and Timiras (1962) found that sexually mature female rats and mice exhibited greater convulsive reactivity than males of the same age and strain, and rhythmic variations in estrogen levels during the estrous cycle were accompanied by parallel variations in brain thresholds. Moreover, the administration of estradiol to intact mature males and to ovariectomized mature and immature females markedly lowered seizure thresholds as a function of dose (Wooley \& Timiras, 1962). At the human level, ovariectomy has been found to reduce the incidence of epileptic seizures. Such seizures often begin, or preexisting attacks often recur or worsen, at menarche; the incidence of seizure is significantly reduced at menopause (Logothetis, Harner, Morrell, \& Torres, 1959). Thus, high levels of estrogens are associated with increased intensity of response activation, a condition which, in Hull's theory, should result in the generation and accumulation of increased levels of $I_{R}$.

The human menstrual cycle provides a convenient context within which endogenous variations in estrogen levels can be explored in relation to reminiscence and its assumed underlying process. Although there is some variation in the timing of the critical events of this cycle, follicular growth-commences around the 8th day of a 28-day cycle in response to the secretion of follicle stimulating hormones (FSH) by the pituitary gland, and the growing follicles begin to secrete estrogens. The estrogens, in turn, stimulate the hypothalamus to cause the production and release of surges of luteinizing hormones $(\mathrm{LH})$ by the anterior pituitary. The $\mathrm{LH}$ surges cause the rupture of a follicle, which then releases an ovum that will shortly become a corpus luteum releasing progesterone. The estrogen level remains relatively low during the first 8 days of the cycle, rises sharply to peak levels around the 14th day (ovulatory phase), subsides moderately for 3 or 4 days, rises again more gradually to a smaller peak accompanied by a rise in progesterone (luteal phase), and finally falls to the level characteristic of the menstrual phase. These facts suggest that the measurement of reminiscence on Days 2,8 , and 14 of a 28-day cycle, or at proportionally corresponding points in longer and shorter cycles, should allow one to measure the effect of maximum variation in estrogen levels unconfounded with variation in progesterone, although it should be pointed out that the FSH and LH levels are also high during the ovulatory phase. However, FSH and LH have never been implicated in the activation function ascribed to estrogens.

Given the foregoing context of the study, two alternatives to the null hypothesis are testable. The first, the "blahs" hypothesis, states that females should reminisce maximally during the menstrual phase (Day 2) because they generally feel uncomfortable and irritable and are thus more susceptible to the effects of massed practice. The second, the "activation" hypothesis, says that females should reminisce maximally during the ovulatory phase (Day 14) because they are more activated by the high estrogen levels and thus, in Hull's theory, generate more $I_{R}$. And, of course, the higher the level of $I_{R}$, the larger the amount of reminiscence.

\section{METHOD}

\section{Subjects}

The experimental sample consisted originally of 45 Caucasian students who volunteered to monitor their menstrual cycles for 2 months and to report for testing on Day 2, 8, or 14 of the cycle, or on proportionally equivalent days for cycles longer or shorter than 28 days. However, two subjects were unable to report for testing on the appointed days. The actual sample, therefore, contained 43 subjects distributed as follows: Day 2 $(n=14)$, Day $8(n=14)$, and Day $14(n=15)$. Ages ranged from 18 to 21 years, with an average of 19.3 years. All subjects were right-handed and normally sighted. None used contraceptive pills and all reported themselves drug free on the day of testing.

\section{Task}

The study employed a mirror tracking task which required subjects to use mirror vision to track a small silver target as it moved clockwise at $1 \mathrm{rpm}$ through a narrow star-shaped pathway (Payne \& Artley, 1972). Subjects were instructed to keep the stylus on target as long as possible and to avoid contact with the sides of the pathway. Time on target was recorded in units of $.01 \mathrm{sec}$ for each minute of practice.

\section{Procedure}

The original 45 volunteers were assigned without bias to three groups (Day 2, 8, or 14 of the menstrual cycle or their proportional equivalents), with the constraint that each group contained 15 subjects. As noted earlier, two subjects failed to keep their testing appointments. Following task instructions, all subjects were exposed to a practice sequence consisting of 3 min massed practice (Trials 1-3), 3 min rest, 3 min massed practice (Trials 4-6), 3 min rest, and $3 \mathrm{~min}$ massed practice (Trials 7-9), as in Huang and Payne (1975). Debriefing concluded the testing session.

\section{RESULTS}

Experimental outcomes were measured in terms of (a) reminiscence scores and (b) performance levels on Trial 4 following their adjustment for regression upon the performance levels on Trial 1.

\section{Reminiscence}

The interpolation of two rest periods in the practice sequence provided for the computation of two reminiscence scores for each subject. The first was found by 
subtracting the score on Trial 3 from the score on Trial 4, while the second was found by subtracting the score on Trial 6 from that on Trial 7. The 86 values were then subjected to a mixed-model variance analysis, in which groups comprised the main effect between subjects and reminiscence periods comprised the main effect within subjects. Neither period effects nor the interaction of Period by Group were significant sources of variation $(\mathrm{F}<1)$. Accordingly, a standard deviation of expected replication differences was derived from the between-subjects error term for use in directional t tests of group mean differences.

The "blahs" hypothesis. This alternative to the null hypothesis was examined by comparing the mean of the Day 2 group (the "blahs" group) with an equally weighted linear combination of the means of the other groups. The means thus compared were 5.9 and 6.26 , respectively $[\mathrm{t}(40)=.26, \mathrm{p}>.05]$. The "blahs" hypothesis was obviously untenable.

The "activation" hypothesis. This comparison involved the mean of the Day 14 group (high estrogen) and an equally weighted linear combination of the means of the Day 2 and Day 8 groups (low estrogen). The means thus compared were 7.97 and 5.17 , respectively $[\mathrm{t}(40)=1.99$, $\mathrm{p}<.05$ ]. The null hypothesis was therefore rejected in favor of the specified alternative.

\section{Performance Level on Trial 4}

The low reliability of reminiscence scores (Eysenck, 1956; Huang and Payne, 1975) and persistent negative correlations between crude gain scores and initial status (Manning \& DuBois, 1962) suggested that experimental treatment effects might be assessed more precisely by a variance-covariance analysis in which scores on the first postrest trial (Trial 4) served as the dependent variable and scores on Trial 1 served as the covariate. Although it is recognized that the experimental treatment might have affected the values of the covariate, there was in fact no significant difference between the three groups on Trial $1[\mathrm{~F}(2,40)<1]$. Accordingly, a standard deviation of expected replication differences was derived from the adjusted error term for use in directional $t$ tests of adjusted group mean differences.

The "blahs" hypothesis. As before, the appropriate comparison was between the adjusted mean of the Day 2 group and an equally weighted linear combination of the adjusted means of the Day 8 and Day 14 groups. The means compared were 23.85 and 25.45 , respectively $[\mathrm{t}(39)=.78, \mathrm{p}>.05]$. Again the "blahs" hypothesis was found untenable.

The "activation" hypothesis. As before, the appropriate comparison was between the adjusted mean of the Day 14 group and an equally weighted linear combination of the adjusted means of the Day 2 and Day 8 groups. The means compared were 28.19 and 23.19 , respectively $[t(39)=2.46, p<.01]$. The null hypothesis was therefore rejected in favor of the specified alternative.

\section{DISCUSSION}

The authors recognize that there was some unknown degree of error in the estimates of estrogenic status and, therefore, also in the decisions concerning the calendar days on which the subjects would be tested. Ideally, of course, estrogen levels might have been monitored frequently by blood and/or urine analyses together with daily temperature measurements throughout the preliminary charting period. These procedures, however, were beyond the authors' logistic capability. Instead, they employed a widely accepted empirical model of the hormonal events of a 28-day cycle (Nocenti, 1968) and made proportional adjustments for subjects who reported longer or shorter cycles. Day 2 of the menstrual period, of course, was estimated without error and with virtual assurance that subjects were at the nadir of the estrogen cycle. With respect to Days 8 and 14, however, estimates of estrogen levels could have been off, on the average, by 1-2 days.

Within the foregoing reservations concerning the values of the independent variable, the experimental data showed quite clearly that subjects at or near the peaks of their estrogen cycles gained more across rest than those at or near the nadirs of their cycles. Since the average female exhibits a more or less elevated estrogen level throughout approximately $70 \%$ of her menstrual cycle (Nocenti, 1968), the chances are about 7 in 10 of drawing a subject in that condition by random sampling from a parent population. Thus, it appears that the estrogen activation hypothesis provides a plausible account of female dominance in reminiscence when the experimental samples are drawn without regard for phases of the menstrual cycle. Viewing matter in somewhat different perspective, one can say that females are most unlike males during the elevated portions of their estrogen cycles, while they are most male-like during the low portions. Mean reminiscence values vary accordingly and compare favorably with those found for the two sexes by Huang and Payne (1975), particularly with regard to males (4.83) vs. low-estrogen females (5.17).

The foregoing conceptualization, of course, should apply as well to the facts of prepubescent male dominance in reminiscence (Horn, 1975; Zegiob \& Payne, 1977). Unfortunately, the empirical status of prepubescent sex differences in gonadal steroid hormone output has not been well established, but the available data have not revealed significant sex differences (Tanner \& Gupta, 1968). However, no large difference in hormone levels would be required because the degree of prepubescent male dominance in reminiscence is much less than the degree of postpubescent female dominance (Payne \& Huang, 1977; Zegiob \& Payne, 1977). As bioassay procedures increase in reliability and precision, perhaps relevant differences will be found. If they are not, one can still suppose that male dominance in reminiscence proclivity is the natural state of affairs and that this relationship becomes obscured by postpubescent estrogenic influences. Whatever the case, it is obvious that much work remains to be done.

\section{REFERENCES}

Ammons, R. B. Acquisition of motor skill: II. Rotary pursuit performance with continuous practice before and after a single rest. Journal of Experimental Psychology, 1947, 37, 393-411.

Arche R, E. J., \& Bourne, L. E., JR. Inverted-alphabet printing as a function of intertrial rest and sex. Journal of Experimental Psychology, 1956, 52, 322-328.

Broverman, D. M., Klaiber, E. L., Kobayashi, Y., \& Vogel, W. Roles of activation and inhibition in sex differences in cognitive abilities. Psychological Review, 1968, 75, 23-50.

Buxton, C. E., \& Grant, D. A. Retroaction and gains in motor learning: II. Sex differences, and a further analysis of gains. Journal of Experimental Psychology, 1939, 25, 198-208.

Eysenck, H. J. Reminiscence, drive, and personality theory. Journal of Abnormal and Social Psychology, 1956, 53, 328-333. 
Gerall, A. A., Napoli, A. M., \& Cooper, U. C. Daily and hourly estrous running in intact, spayed and estrone implanted rats. Physiology \& Behavior, 1973, 10, 225-229.

HonN, P. W. Pursuit rotor speed, sex differences, and reminiscence in young children. Journal of Psychology, 1975, 91, 81-85.

Huang, K. L., \& Payne, R. B. Individual and sex differences in reminiscence. Memory \& Cognition, 1975, 3, 252-256.

Hull, C. L. Principles of behavior. New York: Appleton-CenturyCrofts, 1943.

IrION, A. L. Reminiscence in pursuit-rotor learning as a function of length of rest and of amount of pre-rest practice. Journal of Experimental Psychology, 1949, 39, 492-499.

Kawakami, M., \& Sawyer, C. Effects of hormones and antifertility steroids on brain thresholds in the rabbit. Endocrinology, 1967, 80, 857-871.

Kimble, G. A., \& Horenstein, B. Reminiscence as a function of the amount of interpolated rest. Journal of Experimental Psychology, 1948, 38, 239-244.

Logothetis, J., Harner, R., Morrell, F., \& Torres, F. The role of estrogens in catamenial exacerbation of epilepsy. Neurology, 1959, 9, 352-360.

Manning, W. H., \& DuBois, P. H. Correlation methods in research on human learning. Perceptual and Motor Skills, 1962 , 15, 287-321.

McBride, D. K., \& PAyne, R. B. Psychomotor reminiscence as a function of sex and length of rest period. Journal of Motor Behavior, 1979, 11, 59-64.

McCaffrey, R. J., \& PAyne, R. B. Interaction of sex and practice distribution effects. Bulletin of the Psychonomic Society, 1977, 10, 382-384.
Nocenti, M. R. Reproduction. In V. B. Mountcastle (Ed.), Medical physiology. St. Louis: Mosby, 1968.

Payne, R. B., \& Artuey, C. W. Facilitation of psychomotor learning by classically differentiated supplementary feedback cues. Journal of Motor Behavior, 1972, 4, 47-55.

Payne, R. B., \& Huang, K. L. Interaction of sex and task differences in reminiscence. Journal of Motor Behavior, 1977, 9, 29-32.

Resick, P. A., \& PAYNe, R. B. Sex and practice distribution effects in children. Bulletin of the Psychonomic Society, 1978, 11, 380-382.

Tanner, J. M., \& Gupta, D. A longitudinal study of the secretion of individual steroids in children from 8 to 12 years old. Journal of Endocrinology, 1968, 41, 139-156.

Vogel, W., Broverman, D. M., \& Klaiber, E. L. EEG responses in regularly menstruating women and in amenorrheic women treated with ovarian hormones. Science, 1971, 172, 388-391.

Wooley, D. E., \& Timiras, P. S. The gonad-brain relationship: Effects of female sex hormones on electroshock convulsions in the rat. Endocrinology, 1962, 70, 196-209.

Young, W. C., \& Fish, W. R. The ovarian hormones and spontaneous running activity in the female rat. Endocrinology, 1945, 36, 181-189.

Zegiob, L., \& Payne, R. B. Reminiscence in children as a function of sex. Bulletin of the Psychonomic Society, 1977, 9, 173-175.

(Received for publication June 25, 1979.) 